\title{
Colovesical Fistulae: The Varying Aetiologies
}

Review began 11/19/2021 Review ended 11/27/2021 Published 11/29/2021

\section{() Copyright 2021}

Zafar et al. This is an open access article distributed under the terms of the Creative Commons Attribution License CC-BY 4.0. which permits unrestricted use, distribution, and reproduction in any medium, provided the original author and source are credited.
Mansoor Zafar ${ }^{1}$, Sara Lee ${ }^{2}$, Serena Tieger ${ }^{3}$, William Sacre ${ }^{4}$, Mark Whitehead ${ }^{5}$

1. Gastroenterology and Hepatology, and General Internal Medicine, Conquest Hospital, East Sussex Healthcare NHS Trust, St. Leonards-on-Sea, GBR 2. General Internal Medicine, Conquest Hospital, East Sussex Healthcare NHS Trust, St. Leonards-on-Sea, GBR 3. Internal Medicine, Conquest Hospital, East Sussex Healthcare NHS Trust, St. Leonardson-Sea, GBR 4. Radiology, Conquest Hospital, East Sussex Healthcare NHS Trust, St. Leonards-on-Sea, GBR 5. Gastroenterology, Conquest Hospital, East Sussex Healthcare NHS Trust, St. Leonards-on-Sea, GBR

Corresponding author: Mansoor Zafar, 1mansoorzafar@gmail.com

\section{Abstract}

The most common presenting symptoms of colovesical fistulae (CVF) are pneumaturia and fecaluria. The most important aspect remains not only to investigate the aetiology, and the degree of both severity and complexity, but also the subsequent influence of this on overall management. In a younger population, management usually consists of curative surgery. However, this may not be possible in older patients where surgical candidacy is a genuine concern and a clinical challenge arises relating to pursuing a conservative strategy. We attempted to briefly outline how two patients were managed with a similar nonsurgical approach due to frailty. These cases attempt to highlight the importance of multi-disciplinary specialty input, with a view to optimising patient care.

Categories: Internal Medicine, Medical Education, Anatomy

Keywords: multi-disciplinary team approach, ct-abdomen \& pelvis (portal venous phase), mri fast relaxation fast spin echo sequence), contrast-enhanced ct-abdomen \& pelvis, colo vesical fistulae

\section{Introduction}

A colovesical fistula (CVF) is defined as an abnormal connection between the colon and urinary bladder [1]. The precise incidence of CVF is unknown: it is estimated that they account for 1 in every 3000 surgical hospital admissions [1]. In patients with diverticular disease, 2-18\% were found to have CVF [2-5].

The male-to-female ratio of CVF is approximately 2:1 to 3:1 [6-10]. It has been hypothesised that females are protected from developing CVF due to the presence of the uterus and broad ligaments acting as a barrier between the sigmoid colon and the bladder. This theory is supported by the fact that a high percentage of females with CVF ( $>50 \%$ in some series) have had a previous hysterectomy [11].

We present a case series report of two female patients, with different aetiologies contributing to the development of colovesical fistulae, along with the sequelae.

\section{Case Presentation \\ Case series report}

Case 1

An 83-year-old female was admitted with dysuria, pneumaturia, and malaise. Past medical history included diverticulosis, hypertension, gout, Barrett's oesophagus, recurrent urinary tract infections (UTIs), stable non-Hodgkin's lymphoma, and total abdominal hysterectomy (1983). On examination, baseline observations were stable, and mild suprapubic tenderness was noted. A bedside urine test was positive for nitrites and leukocytes; the urine culture identified Escherichia coli with extended-spectrum beta-lactamase (ESBL) positivity. Admission blood tests showed a mildly raised C-reactive protein (CRP) 32 [5], with a normal white cell count (WCC) of $6.08 \times 109 / \mathrm{L}$ [4-11]. Initial management included intravenous fluid resuscitation and antibiotics.

Given the history of recurrent UTIs and recent pneumaturia, a CT-abdomen and pelvis was performed to exclude a colovesical fistula (Figure 1). Subsequent to this, magnetic resonance imaging (MRI) of the pelvis was undertaken, which demonstrated findings consistent with a colovesical fistula (Figure 2). The case was discussed in a multi-disciplinary meeting with input from clinical specialties including gastroenterology, general surgery, and radiology. Non-surgical management of the CVF with oral antibiotics was the agreed outcome and the patient was discharged with a course of nitrofurantoin. 


\section{Cureus}
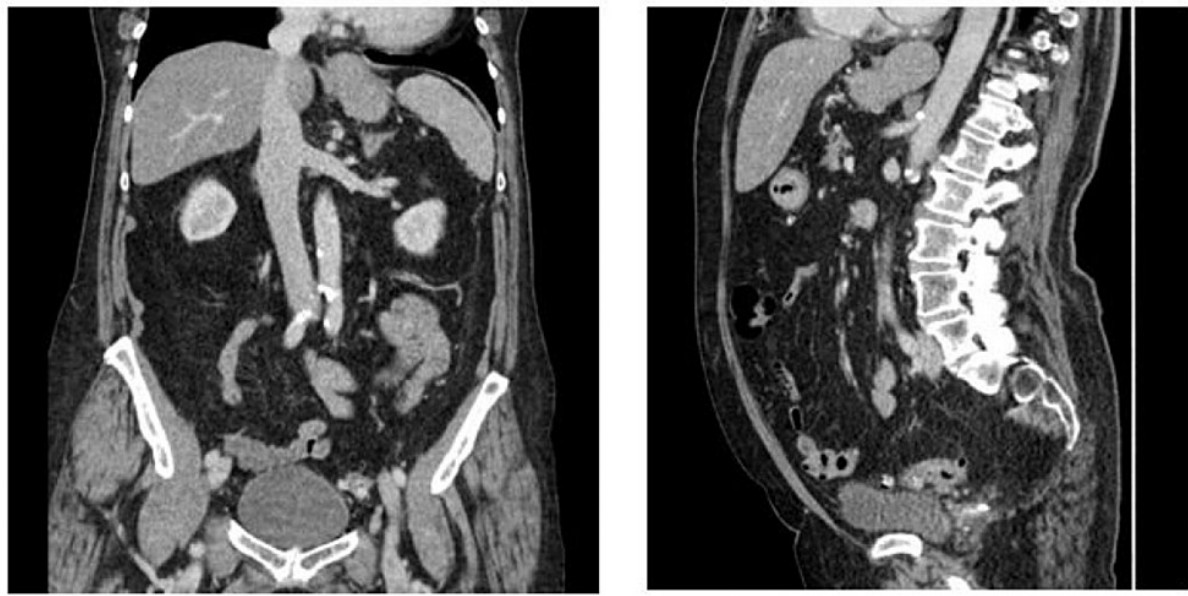

FIGURE 1: Contrast-enhanced CT-abdomen and pelvis (portal venous phase): coronal and sagittal views.

There are no indirect signs or obvious adherence of sigmoid colon to the urinary bladder. However, it is not possible to definitively exclude a fistula on CT and, given the clinical details, an MRI scan may be useful to further evaluate these tissues in more detail.

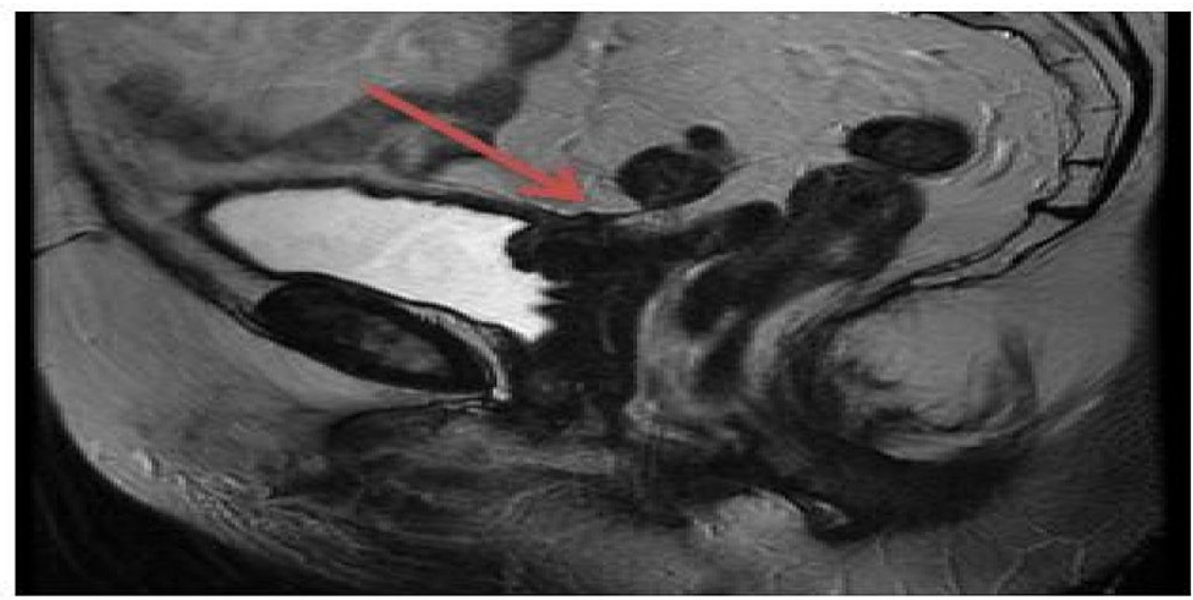

FIGURE 2: MRI-pelvis: sagittal view (T2 fast relaxation fast spin-echo sequence).

Linear area of soft tissue tethering the distal sigmoid colon to a thickened area of the posterosuperior urinary bladder wall. These features likely represent a colovesical fistula.

Case 2

An 88-year-old female presented to the emergency department with acute confusion. Past medical history included a recent diagnosis of type 1 diabetes mellitus, severe frailty, multiple falls, sigmoid diverticulosis. Initial baseline observations were unremarkable. Admission blood tests were essentially unremarkable. A bedside urine test was positive for leukocytes and nitrite; the subsequent culture revealed $>105 / \mathrm{cm}^{3}$ heavy mixed growth. Initial management consisted of intravenous fluids and antibiotics. A CT-head excluded an acute intracranial event. Due to the recent diagnosis of type 1 diabetes mellitus in an older patient, a CTabdomen and pelvis was performed to exclude a pancreatic malignancy. Whilst no concerning pancreatic pathology was identified, a large colovesical fistula was incidentally identified (Figure 3). 


\section{Cureus}
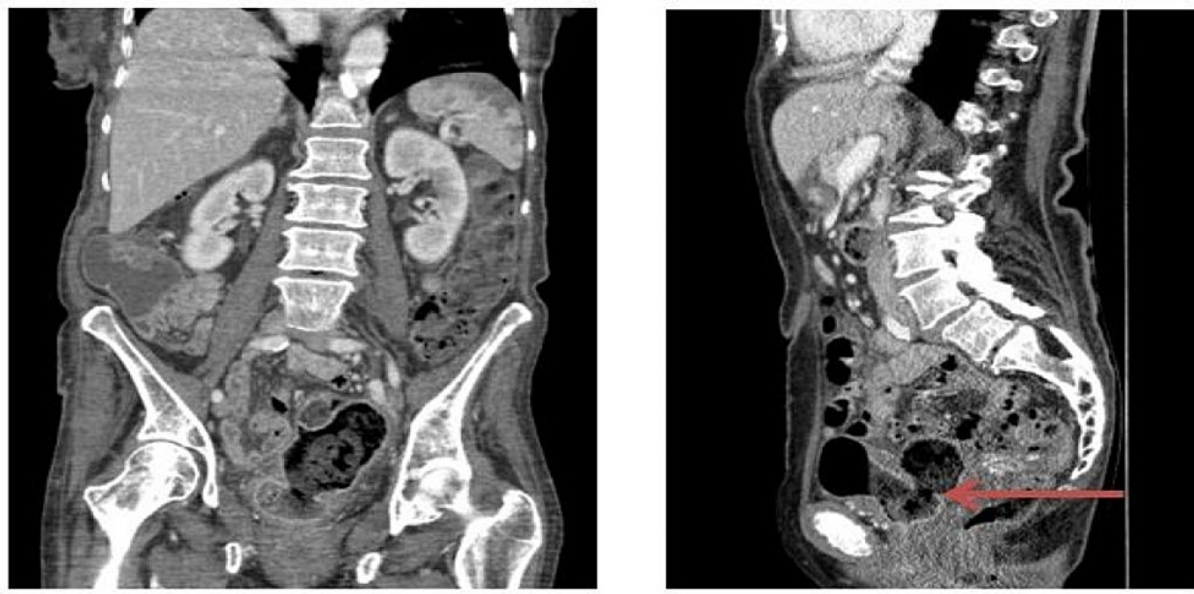

FIGURE 3: Contrast-enhanced CT-abdomen and pelvis (portal venous phase): coronal and sagittal views.

The wide-necked connection between the proximal sigmoid and the bladder with extensive faecal residue filling the bladder, consistent with a colovesical fistula.

The case was discussed in a multi-disciplinary meeting with input from clinical specialties including gastroenterology, general surgery, urology, and radiology. A decision was made in light of her severe frailty for non-surgical management. Following advice from microbiology, alternating prophylactic antibiotic regimes were commenced, rotating every three months. Periodic follow-up in the surgical outpatient clinic was also arranged and the patient was discharged.

\section{Discussion}

The first description of a colovesical fistula has been reported to be attributed to Rufus of Ephesus in AD 200 [12]; however, it was in 1888 that Cripps produced his classic monograph on the subject [11].

A number of causes have been reported to be the aetiology of colovesical fistulae, with the most notorious being a diverticular disease, as well as a sequel to inflammatory bowel disease, more specifically Crohn's disease $[13,14]$. Holmes et al. have reported incidence with colorectal, gynaecological, and urological malignancies $[14,15]$. Subsequent to a foreign body in the gastrointestinal tract [16], laparoscopic and open inguinal hernia repairs [17] and benign gastrointestinal and urological diseases [18,19] have also been reported. Kiani et al. conducted a comprehensive study at Guy's and St. Thomas' NHS Trust of 55 patients with a diagnosis of CVF. They concluded that although having a similar clinical presentation, colovesical fistulae of various aetiologies differ significantly in management and outcome [19].

We attempted to highlight the case series report of two patients. One case had the usual sequel including a background history of abdominal hysterectomy. The second case presented with no appropriate cause or iatrogenic background, presenting as a clinical challenge. Both patients were managed in a similar way following a conservative approach due to frailty and patient autonomy. This emphasizes that patients can be managed conservatively in cases where surgical intervention may not be appropriate.

\section{Conclusions}

Colovesical fistulae can occur with or without previous history of the bowel, bladder, or gynaecological procedures. Whilst surgical input is justified in the younger population, management should be decided on a case-by-case basis, with a non-surgical approach being suitable among a frailer populace. Utilisation of imaging together with a multi-disciplinary specialty approach within secondary care permits a patientcentered approach to CVF management.

\section{Additional Information}

\section{Disclosures}

Human subjects: Consent was obtained or waived by all participants in this study. Conflicts of interest: In compliance with the ICMJE uniform disclosure form, all authors declare the following: Payment/services info: All authors have declared that no financial support was received from any organization for the submitted work. Financial relationships: All authors have declared that they have no financial relationships at present or within the previous three years with any organizations that might have an interest in the submitted work. Other relationships: All authors have declared that there are no other 
relationships or activities that could appear to have influenced the submitted work.

\section{Acknowledgements}

We are very thankful to the Department of Gastroenterology and Radiology at Conquest Hospital, East Sussex Healthcare NHS Trust, UK, for their support.

\section{References}

1. Pu JI: On the pathology and behaviour of acquired non-traumatic vesico-intestinal fistula . Br J Surg. 1964, 51:644-57. 10.1002/bjs.1800510903

2. Pollard SG: Colovesical fistula. Ann R Coll Surg Engl. 1988, 70:184.

3. Amin M, Nallinger R, Polk HC Jr: Conservative treatment of selected patients with colovesical fistula due to diverticulitis. Surg Gynecol Obstet. 1984, 159:442.

4. Menenakos E, Hahnloser D, Nassiopoulos K, Chanson C, Sinclair V, Petropoulos P: Laparoscopic surgery for fistulas that complicate diverticular disease. Langenbecks Arch Surg. 2003, 388:189-93. 10.1007/s00423003-0392-4

5. Labs JD, Sarr MG, Fishman EK, Siegelman SS, Cameron JL: Complications of acute diverticulitis of the colon: improved early diagnosis with computerized tomography. Am J Surg. 1988, 155:331-6. 10.1016/s00029610(88)80726-8

6. Smeenk RM, Plaisier PW, van der Hoeven JA, Hesp WL: Outcome of surgery for colovesical and colovaginal fistulas of diverticular origin in 40 patients. J Gastrointest Surg. 2012, 16:1559-65. 10.1007/s11605-0121919-1

7. Morrison PD, Addison NV: A study of colovesical fistulae in a district hospital . Ann R Coll Surg Engl. 1983, $65: 221-3$.

8. Holroyd DI, Banerjee S, Beavan M, Prentice R, Vijay V, Warren SI: Colovaginal and colovesical fistulae: the diagnostic paradigm. Tech Coloproctol. 2012, 16:119-26. 10.1007/s10151-012-0807-8

9. Woods RJ, Lavery IC, Fazio VW, Jagelman DG, Weakley FL: Internal fistulas in diverticular disease . Dis Colon Rectum. 1988, 31:591-6. 10.1007/BF02556792

10. Kellogg WA: Vesico-enteric fistula. Am J Surg. 1938, 41:135-86.

11. Cripps H: Passage of air and faeces from the urethra. Lancet. 1888, 2:619.

12. Rao PN, Knox R, Barnard RJ, Schofield PF: Management of colovesical fistula. Br J Surg. 1987, 74:362-3. 10.1002/bis.1800740511

13. Garcea G, Majid I, Sutton CD, Pattenden CJ, Thomas WM: Diagnosis and management of colovesica fistulae; six-year experience of 90 consecutive cases. Colorectal Dis. 2006, 8:347-52. 10.1111/j.14631318.2005.00928.x

14. Holmes SA, Christmas TJ, Kirby RS, Hendry WF: Management of colovesical fistulae associated with pelvic malignancy. Br J Surg. 1992, 79:432-4. 10.1002/bjs.1800790520

15. Khan MS, Bryson C, O'Brien A, Mackle EJ: Colovesical fistula caused by chronic chicken bone perforation . Ir J Med Sci. 1996, 165:51-2. 10.1007/BF02942805

16. Daoud F, Awwad ZM, Masad J: Colovesical fistula due to a lost gallstone following laparoscopic cholecystectomy: report of a case. Surg Today. 2001, 31:255-7. 10.1007/s005950170181

17. Abbas F, Memon A: Colovesical fistula: an unusual complication of prostatomegaly . J Urol. 1994, 152:47981. 10.1016/s0022-5347(17)32770-2

18. Cockell A, McQuillan T, Doyle TN, Reid DJ: Colovesical fistula caused by appendicitis . Br J Clin Pract. 1990, 44:682-3.

19. Kiani QH, George ML, Carapeti EA, Schizas AM, Williams AB: Colovesical fistula: should it be considered a single disease?. Ann Coloproctol. 2015, 31:57-62. 10.3393/ac.2015.31.2.57 